\title{
Ein historischer Beitrag zur Methodologie der biologischen Wissenschaften
}

\section{Kommentar zu Ilse Jahn: Matthias Jacob Schleiden an der Universität Jena (1963)}

Dietrich von Engelhardt

In ihrer Studie behandelt die vor wenigen Wochen verstorbene Biologiehistorikerin Ilse Jahn (1922-2010), ebenso bekannt wie geschätzt durch ihre weitgespannten, differenzierten und stets quellennahen Arbeiten zur Geschichte der Biologie und vor allem auch als Herausgeberin der Geschichte der Biologie. Theorien, Methoden, Institutionen, Kurzbiographien (1982, 3. Aufl. 1998, erneut 2004), eine wesentliche Phase in der Vita eines bedeutenden Naturforschers des 19. Jahrhunderts.

Die Beziehungen zwischen Philosophie und Naturwissenschaft - wie auch Medizin - waren um 1800 überaus komplex und vielfältig. Kant (1724-1804), Schelling (1770-1831) und Hegel (1775-1854) fanden Resonanz und waren selbst bestens vertraut mit dem Stand der naturwissenschaftlichen und medizinischen Disziplinen ihrer Zeit. Der transzendentalen Naturphilosophie Kants stand die metaphysische Naturphilosophie gegenüber, in der spekulativen Gestalt Schellings und Hegels sowie der romantischen Form zahlreicher Naturforscher und Mediziner. Von diesen 
naturphilosophischen Positionen unterschied sich wiederum eine empirische Naturforschung und entsprechende Wissenschaftstheorie und Methodologie der Forschung. Die Entwicklung der Naturwissenschaften und Medizin ist keineswegs insgesamt - auch in Deutschland nicht - durch eine Phase der transzendentalen oder metaphysischen Naturphilosophie hindurchgegangen.

Faktische und normative Urteile über die naturwissenschaftliche Bedeutung von Matthias Jacob Schleiden (1804-1881) entfalteten sich vor diesem weitgespannten Spektrum, das in seinen Grundzügen, Überschneidungen und Unterschieden nicht im Zentrum dieser Studie steht, und fielen auch bereits zu Lebzeiten von Schleiden kontrovers aus (zwischen Kritik der falschen Zellbildungslehre und Anerkennung als Entdecker der Zelle). Mit seinem induktiven Wissenschaftsverständnis, seinem methodischen Programm und seinen empirischen Forschungen zur Entstehung und Funktion der Zelle trug Schleiden, wie Ilse Jahn überzeugend ausführt, sowohl wesentlich zur Entwicklung der modernen Pflanzenphysiologie als auch der pharmazeutischen und medizinischen Praxis bei. Jahns besonderes und innovatives Interesse gilt in dieser knappen Studie von zehn Seiten der wissenschaftstheoretischen Position Schleidens, den Voraussetzungen und Folgen, der Opposition zur metaphysischen Naturphilosophie, die bekanntlich nicht zuletzt in Jena in den Naturwissenschaften und der Medizin eine große Rolle gespielt hat. Persönliche Verbindungen zu Wissenschaftlern an diesem Ort waren vielfältig gegeben. Prägend für seine wissenschafts- und erkenntnistheoretische Position waren die Philosophie von Jacob Friedrich Fries - Anhänger Kants, Gegner Schellings und Hegels - und die Einflüsse von Naturforschern und Medizinern in Göttingen und Berlin, worauf mit vielen Details und biographischen Hinweisen von Ilse Jahn eingegangen wird. Wesentliches Gewicht schreibt sie in diesem Zusammenhang dem englischen Botaniker Robert Brown (1773-1858) zu, den Schleiden für ein ,botanisches Genie“ hielt und als Entdecker des Zellkerns mit großer Anerkennung in den Beiträgen zur Phytogenesis (1838), den Grundzügen der wissenschaftlichen Botanik (1842/ 43) sowie auch in seiner Vita von 1839 mehrfach preist.

Wissenschaftsgeschichte ist Geschichte von Ideen, Theorien, Methoden, Kenntnissen, aber stets auch Geschichte von Institutionen und Personen im sozialkulturellen Kontext, keineswegs immer unabhängig oder unbeeinflusst von ideologischen und politischen Verhältnissen. Das breite Spektrum zwischen Anhängern, Mitläufern, Indifferenten und Gegnern war auch in der Medizinund Wissenschaftsgeschichte der DDR gegeben; eine differen- 
zierte und angemessene, nicht moralisierende und selbstgerechte Analyse steht noch aus. Zahlreich sind die Beispiele und Dokumente der Unabhängigkeit und Freiheit, aber auch der behinderten oder gebrochenen Lebensentwicklungen. Die Deutsche Akademie der Naturforscher Leopoldina, jetzt Nationale Akademie der Wissenschaften, in die auch Ilse Jahn 1986 als Mitglied aufgenommen wurde, konnte von ihrem früherem Präsidenten Benno Parthier im Rückblick als „Insel im roten Meer" bezeichnet werden. Im Übrigen muss marxistische Überzeugung auch nicht schon Rechtfertigung von Unrecht heißen. Ilse Jahns Studie über Schleiden lässt jedenfalls keinerlei ideologische Einflüsse jener Epoche erkennen. Profil und Bedeutung der 1960 in Leipzig gegründeten NTM motivierten die neuen Herausgeber nach der Wende zur erfolgreichen Weiterführung dieser Zeitschrift.

Ilse Jahn geht auf Schleidens Initiativen in der Forschung und Lehre ebenso wie auf psychologische Krisen ein, an denen der Naturforscher während seiner juristischen Tätigkeit zu leiden hatte und die ihn auch an Selbstmord denken ließen, bevor er sich für die Medizin und die Naturwissenschaften und vor allem Botanik entschied und 1839 nach Jena kam. Die Verbindung von Vita und Werk wurde in der Epoche um 1800 allgemein für wesentlich gehalten, jedoch bislang von der Wissenschaftsgeschichte kaum behandelt. Offensichtlich ist aber für Entstehung und Ausbreitung der Wissenschaften das Zusammenwirken von Objektivität und Subjektivität, von Empirie und Biographie, von interner Dynamik und externem Kontext.

Goethe (1749-1832) kritisierte Newton (1643-1727) nicht nur wissenschaftlich wegen seiner Theorie der Farben, sondern auch moralisch als starren Charakter: „Das Hauptfundament des Charakters ist das entschiedene Wollen, ohne Rücksicht auf Recht und Unrecht, auf Gut und Böse, auf Wahrheit oder Irrtum. " Hegel erkannte in der Melancholie des Mediziners Carl Josef Hieronymus Windischmann (1775-1839) eine grundsätzliche Phase in der Entwicklung eines jedes Menschen: „Wendungspunkt im Leben“, „nächtlicher Punkt der Kontraktion seines Wesens, durch dessen Enge er hindurchgezwängt und zur Sicherheit seiner selbst befestigt und vergewissert" wird. Der Naturforscher Henrik Steffens (1773-1845) blickte mit Zustimmung auf sein vergangenes Leben zurück: „So bin ich bereit, das Leben zu verlassen, wie ich früher mein Vaterland verließ. Die um mich herrschende Verwirrung stört mich nicht, und meine jugendliche Hoffnung liegt vor mir; sie ist nicht eine abstracte von mir getrennte: sie ist im vollsten Sinne Meine." Der Dichter Clemens Brentano (1778-1842) klagte 
dagegen zu Ende seines Lebens: „Wir sind Nichts mehr, wir gelten Nichts, wir wissen nicht mehr, wer wir sind, ahnen kaum, wer wir waren."

Schleiden, der sich selbst von „keiner sittlich religiösen Lebensansicht" und „wahrem Vertrauen“ zu einer anderen Person getragen fühlte, erhielt in der Philosophie von Fries die gesuchte theoretische Orientierung und den notwendigen individuellen Halt. Mit Recht weist Ilse Jahn für die Jenenser Zeit auf Schleidens Vorlesungen „Philosophische Botanik“, „Über den Gebrauch des Mikroskops" und "Physiologia comparata" und die Einrichtung eines physiologischen Praktikums hin, aus dem später das Physiologische Institut hervorging, bedeutsam nicht nur für den wissenschaftlichen Fortschritt, sondern ebenfalls den experimentellen Unterricht der Medizinstudenten. Auch an der Gründung der Zeitschrift für wissenschaftliche Botanik war Schleiden ideell über die Namensgebung beteiligt. In der Überzeugung, dass es nur „Eine Natur und Eine Wissenschaft von derselben“ gebe, zeigt sich eine Nähe Schleidens, der auch künstlerisch produktiv war, zu der von ihm vehement kritisierten Naturphilosophie und Romantik.

Weitreichend sind die Auswirkungen von Schleidens Leistungen auf die biologischen Wissenschaften und besonders die Botanik mit einer Vielzahl neuer Fragestellungen und Forschungsaufgaben, mit seinen Beiträgen zum Verständnis der Zelle und Zellbildung sowie der Befruchtungsvorgänge, mit seinem Schwerpunkt in der mikroskopischen Anatomie, mikroskopischen Pharmakognosie, seinem Eintreten für experimentelle pflanzenphysiologische Methoden und stets auch praktisch-industrielle Einrichtungen.

Anregend ist die Studie von Ilse Jahn über Matthias Schleiden in seiner Zeit in Jena nicht nur in den Beobachtungen und Interpretationen, sondern ebenso in den Fragen, zu denen sie zukünftige Forschungen zu stimulieren weiß: Einordnung in das Spektrum der Positionen zwischen Philosophie und Wissenschaft der Natur um 1800, Zusammenhang von Leben und Werk, Verhältnis von theoretischer Auffassung und praktischer Umsetzung, Beziehung von Forschung und Lehre, künstlerische Produktion eines Naturwissenschaftlers. Ilse Jahn ist selbst diesen Fragen in späteren Arbeiten und vor allem gemeinsam mit ihrer Tochter Isolde Schmidt in der Monographie Matthias Jacob Schleiden (1804-1881). Sein Leben in Selbstzeugnissen (2005) auf eindrucksvolle Weise nachgegangen. 
Ein historischer Beitrag zUr Methodologie der biologischen Wissenschaften

Dietrich von Engelhardt

Institut für Geschichte und Ethik der Medizin

Ismaninger Strasse 22

81675 München

Deutschland

E-Mail: v.e@imgwf.uni-luebeck.de 